\title{
Using Location Information for Scheduling in 802.15.3 MAC
}

\author{
Sethuram Balaji Kodeswaran, Anupam Joshi \\ Department of Computer Science and Electrical Engineering \\ University of Maryland Baltimore County \\ 1000, Hilltop Circle, Baltimore, MD 21250 \\ Email: (kodeswar, joshi)@cs.umbc.edu
}

\begin{abstract}
In recent years, UWB has received much attention as a suitable Physical Layer (PHY) for Wireless Personal Area Networks (WPANS). UWB allows for low cost, low power, high bandwidth, short reach communication well suited for personal operating spaces. One of the key features offered by UWB is very accurate ranging between a transmitter/receiver pair. The IEEE 802.15.3 is a MAC protocol that has been proposed for WPANs. In this MAC, a combination of CSMA/CA and TDMA is used to achieve channel scheduling. The TDMA component ensures only one transmitter/receiver pair within a piconet is active at any given time thereby ensuring an exclusion region that covers the whole piconet. In this paper, we propose a less stringent scheduling mechanism that allows for concurrent communication between UWB transmitter/receiver pairs within a piconet. Exclusion is necessary only when the communicating entities are close enough such that interference between them would adversely affect successful reception of data at the receivers. The Piconet Coordinator (PNC) uses the ranging information provided by UWB to accurately position transmitters and receivers. The PNC schedules parallel transmissions between distinct transmit/receive pairs as long as they do not interfere. We present the results of our simulations of our proposed modifications and show that the network throughput can be significantly increased with very little change to the 802.15.3 MAC.
\end{abstract}

\section{INTRODUCTION}

A Wireless Personal Area Network (WPAN) is a network of wireless devices that can communicate over short distances. It is envisioned that WPANS will soon see wide spread use in a multitude of applications like interconnecting home audiovideo appliances, interconnecting computer peripherals and gaming consoles, automatic data synchronization for portable personal devices, interconnecting medical equipment, asset tracking etc. Bluetooth [1] has seen wide acceptance in many consumer appliances such as PDAs and cell phones in recent years as a viable cable replacement technology. However, issues such as limited data rate and interference due to its operation in the unlicensed $2.4 \mathrm{Ghz}$ band restrict the spectrum of applications that can be supported by Bluetooth. Emerging applications require high-speed links to be established between communicating devices and at the same time, enforce constraints such as low power consumption, low cost, low complexity, etc.

Ultra Wide Band (UWB) is an emerging wireless technology that can support high data rates over short distances. Unlike traditional narrowband radio, a UWB radio transmission is characterized by its broad spectrum usage. The FCC defines a UWB radio signal as one with the fractional bandwidth of at least 0.20 (where fractional bandwidth is the ratio of the signal's bandwidth to the signal's center frequency) or has a spectral bandwidth of at least $500 \mathrm{MHz}$. Radiated powers of a UWB transmission is strictly regulated as the spectrum used could overlap with other licensed bands (the FCC has set a limit of $-41.3 \mathrm{dBm} / \mathrm{MHz}$ EIRP in the 3.1 to 10.6 GHz frequency range). This limits the range of UWB transmissions to around $10 \mathrm{~m}$ (greater range is possible at the expense of data rate), which is ideal for communications confined to a personal operating space (POS). UWB exhibits excellent multipath immunity making it a good candidate for indoor WPANS. It also offers immunity against external RF interference and its low power spectral density (PSD) and noise like spectra prevent UWB devices from interfering with other conventional RF devices. The low PSD allows UWB devices to operate covertly providing for low probability of detection and Interception (LPD and LPI) making them good candidates for secure military and commercial applications. In addition, due to its large bandwidth, UWB also allows for the determination of very accurate ranging information that can be used for positioning.

The IEEE 802.15.3a task group [2] has been working towards amending the 802.15.3 [3] standard to serve as the MAC/PHY standard for UWB WPANs. The 802.15.3 MAC was originally designed for a narrowband radio operating in the unlicensed $2.4 \mathrm{GHz}$ spectrum. As a result, features are built into the MAC that are suitable for narrowband radio. The MAC protocol uses a combination of CSMA/CA and TDMA to schedule channel access to communicating devices in a piconet. The goal is to ensure that simultaneous transmissions do not occur within a network, as this will cause collisions and packet loss. This enforces an exclusion region that spans the whole piconet. Such a stringent radio access mechanism prevents interference at the cost of network throughput.

In this paper, we propose an enhancement to the TDMA component of the 802.15.3 MAC that is less stringent and allows for parallel communication between devices within a piconet. Parallel transmissions can occur as long as they do no interfere with each other. We are utilizing the ranging information provided by a UWB PHY to accurately position devices within a piconet. This allows the PNC to schedule non-interfering transmissions to occur concurrently during the 


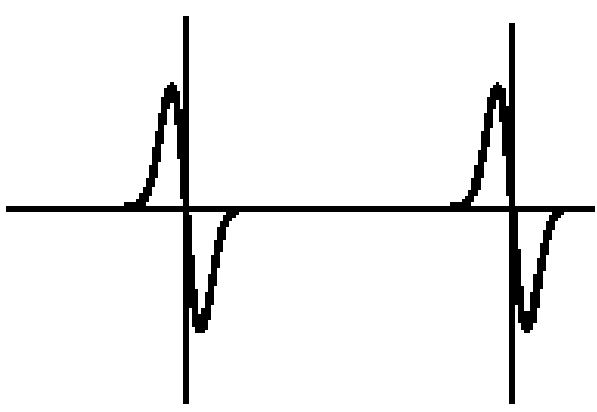

Fig. 1. Monopulses

TDMA phase of the 802.15.3 MAC. The position information is periodically updated to ensure that the possibility of interference is minimized. This allows the throughput of the network to be improved with minimal modification to the MAC. We present the results of simulation studies that we have done that show the benefits that can be achieved through our proposed approach.

The rest of the paper is organized as follows. Section 2 presents existing work dealing with MAC and location related issues in UWB networks. Section 3 presents the network model that we are considering. Section 4 presents the details on the enhancements we are proposing. Section 5 describes the simulation studies that we have conducted to study the effectiveness of our proposed enhancements. Section 6 concludes the paper.

\section{UWB BACKGROUND AND RELATED WORK}

There are essentially two models for the UWB PHY that are being considered for standardization by the IEEE. The DSUWB [4] proposal utilizes direct sequence spreading of BPSK (or optionally 4-BOK) UWB pulses. The available spectrum is split into a lower band $(3.1 \mathrm{GHz}$ to $4.85 \mathrm{GHz})$ and an upper band $(6.2 \mathrm{GHz}$ to $9.7 \mathrm{GHz})$ and within each band, 6 piconet operating channels are defined, each with its specific operating frequencies and acquisition codes. By varying the length of the spreading code used, different data rates can be realized raging from 28Mbps to $1320 \mathrm{Mbps}$. Acquisition sequences used within a piconet for receiver synchronization are encoded using well defined Piconet Access Codewords that allow for separation of overlapping piconets. DS-UWB uses a series of short gaussian monopulses (Fig.1) with a duration of a few nanoseconds for data transmission. A rake receiver is used to resolve the different multipath components of the received signal and by adding them up, the signal-to-noise ratio can be improved. The pulse repetition interval is chosen so that there is enough seperation between them so that the inter symbol interference is avoided.

The other model that is under consideration is the multiband Orthogonal Frequency Division Multiplexing scheme (MOFDM) [5]. The unlincensed spectrum from 3.1 to 10.6 Ghz is divided into 528Mhz bands and these bands are aggregated into 5 band groups (4 bandgroups of 3 bands each

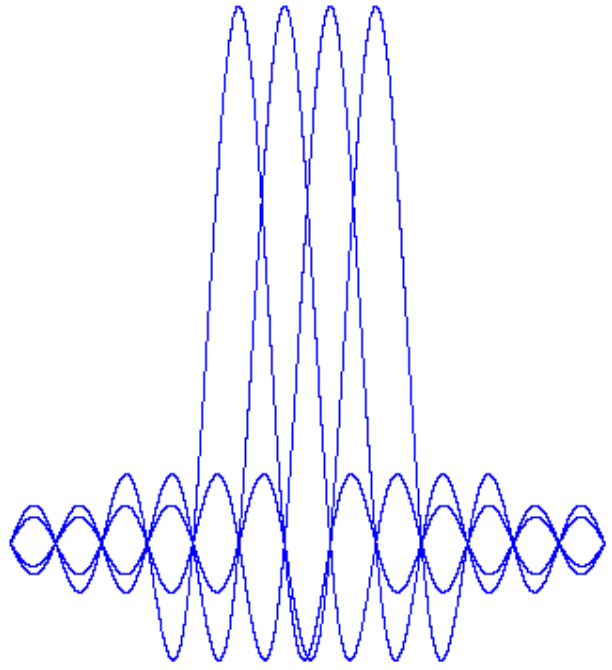

Fig. 2. Orthogonal Frequencies

and 1 bandgroup with 2 bands). The data is interleaved over the bands in a bandgroup using time frequency codes. By varying the coding rates and the frequency-domain spreading factor, data rates varying from 53.3 Mbps to $480 \mathrm{Mbps}$. Unlike DS-UWB, MOFDM transmits data simulataneously across multiple carriers using QPSK. The carriers are chosen so that they are mutually orthogonal to each other so that they do not interfere (Fig.2). At the transmitter, IFFT is used to convert the orthogonal frequencies to a time domain waveform to be transmitted. An FFT is applied at the receiver to convert back to the frequency domain and obtain the transmitted data.

The IEEE 802.15.3 [3] is the MAC standard proposed for high speed WPANs. It was originally defined for a 2.4 $\mathrm{GHz}$ narrowband PHY and the IEEE 802.15.3a task group [2] is tasked with extending this MAC to support the UWB PHY. The network model considered is an ad-hoc collection of devices grouped into small piconets, where each piconet spans a range restricted by personal operating space limits and is controlled by a designated Piconet Coordinator (PNC). A PNC provides the basic timing for the piconet through periodic beacons. The PNC performs admission control for devices intending to join a piconet, schedules media access to all devices that are already part of the piconet and controls the power save mode for these devices. To support coexistence of overlapping piconets, child and neighbor piconets are defined. Devices including the PNC can join and leave a piconet dynamically (in case an existing PNC desires to leave, a handoff occurs and another device in the piconet becomes the PNC). A combination of CSMA/CA (during the contention period) and TDMA (during the contention free period) are used for medium access. The contention period allows for asynchronous data transmission and piconet management commands (device association, request for time slots etc). The TDMA component allows the MAC to provide QoS guarantees that are essential for multimedia applications. The PNC allocates durations during the contention free period 


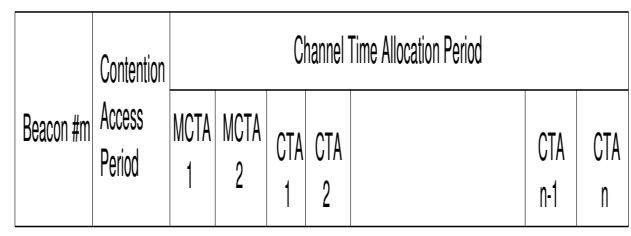

Fig. 3. Superframe Structure

of a superframe (time between consecutive PNC beacons) to requesting devices such that at any given time, only one device can be in transmit mode and one or more devices can be in receive mode thereby preventing possible channel collisions through temporal exclusion. The PNC beacons also serve to time synchronize all devices in the piconet and notify constant or temporary changes to the piconet participants which can be extended through application specific Information Elements (IE). Four different acknowledgement modes namely, no ack, immediate ack, delayed ack and implied ack are specified. Service discovery mechanisms are also built into the protocol to allow devices to register and query for availble services in the piconet. Several interference mitigation mechanisms are built into the MAC with the PNC performing regular channel scans and taking corrective action such as switching to a different channel, reducing the transmit power (thereby limiting the extent of the piconet) or in case of interference from a nearby piconet, forming a child or neighbor piconet relationship between the two to allow each piconet to perform channel allocation without affecting the other. In addition, a pair of devices communicating during their assigned timeslots can negotiate transmit power levels (not exceeding the maximum allowed transmit power within the piconet) to use based on their received powers and localized interference.

Several alternate MAC layers have been proposed for a UWB based networks. The DCC-MAC [9] takes into account Pulse Position Modulation (PPM) [19] UWB specific characteristics and is a decentralized protocol that is intended for low-power, low rate UWB communication. The DCCMAC relies on dynamically adapting the channel code to mitigate interference and enforcing exclusion only around a receiver. This allows parallel transmissions to occur in a network and the bit rates of these transmissions automatically adapt depending on the level of interference between them. A "private MAC" feature is provided to handle contention between sources sending to the same destination. With regards to power control, a source either transmits at maximum power or is silent. This approach has been proposed as a viable alternative to 802.15.4 low power, low rate UWB networks. For high rate networks, however, a rate adaptive MAC is not suitable as strict guarantees in terms of bandwidth and delay are required to support applications such as streaming video. While transmitting at full power will have the benefit of increasing the resiliency against interference from external sources, it incurs the penalty of allowing only one transmission to occur at any given time within the piconet. This limits the number of flows that can be admitted in a network while still being able to meet the bandwidth and delay requirements of each of these flows.

In [12], guidelines for a MAC to support an impulse radio based UWB are discussed. In their model, the data rate is inversely propotional to the chip period, number of chips per pulse repetition period and the number of pulses that need to be integrated at the receiver to identify the transmitted bit. Power control is used to improve spatial reuse and reduce interference between transmitting devices. They present joint power and rate assignment as an optimization problem and consider possible solutions for traffic that is dynamic and traffic that is reserved with guaranteed bandwidth or delay thresholds. Sub-optimum algorithms are presented where medium access is controlled in a distributed fashion using a combination of rate and power control. An interesting observation from this work is that for elastic flows without strict bandwidth or delay requirements, the optimal approach is to use a simple on/off scheme where a transmitter is transmitting at full power or not transmitting at all. However, for supporting QoS constrained reserved flows, a combined power and rate control scheme is more optimal. For a flow to be admitted, the piconet must be able to acheive the desired QoS without adversely affecting the other flows in the network and at the same time, not exceeding the maximum allowable transmit power. In our proposed approach, we exploit the centralized nature of 802.15.3 to schedule parallel flows so that similar constraints are satisfied through power control mechanisms. By limiting the transmit power of sources within a network (to the minimum required power for successful transmission), better spatial reuse of the medium is achieved allowing us to have multiple flows in progress at the same time with no interference between them.

In [15], the authors propose a MAC scheme called the Sustained Link Networks (SLN), that addresses the long acquisition times that are a common problem with impulse radio based networks. Unlike conventional narrowband radio where physical layer links are terminated when there is no data packet for transmission, SLN maintains the physical layer links for the lifetime of the logical link between any two nodes. When there are no data packets to be sent, the physical links are maintained by exchanging periodic control information in small packets at low bit rates and power levels. This minimizes the need for signaling packets containing long acquisition headers that need to be transmitted, usually at a high power thereby improving the covertness offered by UWB making it more attractive for military applications. In this scheme, the transmission power levels are dynamically adjusted to ensure that a desired signal to noise ration is maintained at a receiver. This is achieved through a feedback mechanism between a transmitter and receiver pair. [17] also addresses the problem of long acquisition times but with the aim of reducing the impact of the acquisition times on the performance of high rate links. Instead of sending each upper layer packet in individual MAC frames, the proposed solution aggregates several upper layer frames into a single larger burst frame at the MAC layer. It is shown that this helps to reduce the synchronization 
overhead.

One of the key features offered by UWB as an offshoot of its relatively large bandwidth is very accurate ranging between a transmitter and a receiver. UWB has often been used to implement precision asset tracking systems. Several techniques based on Time of Arrival (TOA), Time Difference of Arrival (TDOA), Angle of Arrival (AoA) and Received Signal Strength (RSS) have been proposed for geo-positioning in indoor environments using UWB. In [16], a TOA based UWB ranging scheme is presented where a direct path signal is detected in dense multipath using a generalized maximumlikelihood estimation technique. The transceivers used accomplish two-way ranging between them without the presence of any common clock. In [10], TOA is estimated for the first multipath and by averaging over multiple time frames, accuracy close to the Cramer-Rao lower bound can be achieved. The assumption made is that there is LOS paths between the transmitter and receiver so that the strongest path is the first multipath and there is perfect synchronization between the transmitter and receiver. In [13], the authors present an integrated UWB transceiver called localizers that allows for rapid positioning and data communication within a network. Localizers exchange specially coded UWB impulses to accurately determine range information with neighors. Clusters of local nodes are formed and information is exchanged across clusters so that all localizers are aware of all other localizers in the network with positional accuracy close to a centimeter. In [14], a prototype precision asset location system is described using a modified version of TOA technique. Using reference nodes whose position is well known within an enviroment, it is possible to use the Euclidean distance between a UWB device and several of these references to extrapolate the physical location of a device. Relative location techniques can be used to improve the accuracy of the location estimate [11].

In this paper, we are proposing augmenting the 802.15.3 MAC to utilize the location information for piconet devices during the superframe scheduling. Using this information, the PNC can perform more efficient scheduling of the medium access and can permit concurrency since the PNC can now decide whether concurrent transmissions will collide or not using their respective location and power of transmission. As shown in the following sections, this will improve the throughput of the piconet and the average packet delay from a transmitter to a receiver. Also, since the anticipated indoor WPAN applications for UWB do not involve too much mobility (and even in these cases, at low speeds), the location updates for the devices within the piconets are not required to happen very frequently keeping the overhead to a minimum.

\section{NETWORK MODEL}

The network model considered in this paper comprises of an indoor environment with devices participating in a WPAN similar to application scenarios such as conference rooms, a home entertainment room etc. We assume the existence of strategically placed UWB reference nodes within this environment. The locations of these nodes are well known apriori and are with respect to some predefined coordinate axis (for example, the center of the room could be defined as $(0,0)$ and the location of these references are with respect to this). Through this, the relative location of devices within a piconet with respect to the coordinate system followed by the reference nodes can be determined. The actual mechanism used to derive the relative location can be any of the commonly used schemes described above and does not impact the enhancement that we are proposing. Devices can enter and leave the environment dynamically. Within the environment, considering the application scenarios proposed, we do not anticipate devices to move around frequently. This implies that the mechanisms used to position nodes in the network do not need to frequently update the position information thereby keeping the overhead to a minimum. We assume that the applications on the mobile nodes have strict QoS requirements in terms of bandwidth and tolerable packet delays. All nodes use 802.15.3 as their underlying MAC protocol.

With the recent advances in the commercialization of UWB chipsets, we believe environments such as the one we are considering are quite feasible in the very near future. The WiMedia alliance [6] has been working to develop a protocol adaptation layer to support Wireless USB and Wireless IEEE1394 (Firewire) over UWB which are likely to see widespread deployment to consumer electronics interconnecting home or office audio/video equipment. Also, UWB localizers are now available in small form-factors making it possible to embed such devices in walls to create smart environments such as the one the we have outlined above.

\section{PROPOSED APPROACH}

In our proposed approach, a device intending to establish a UWB network connection will first scan for an active piconet as specified in the 802.15.3 MAC. Typically in environments such as the one described above, one of the reference nodes can also act as the designated PNC. This will ensure that there is always a piconet available that new devices can associate with and minimizes the overhead of PNC handover as devices leave the environment. The new device upon detecting an existing piconet will initiate a device association process as specified in the 802.15.3 MAC. For this, the contention access period (if allowed in the beacon) or a Management Channel Time Allocation (MCTA) for association may be used. Once admitted, for our enhancement, the PNC needs to know the location of the devices in the piconet. This information can be determined by the PNC by gathering the range information from the other reference nodes. For this, special MCTA allocations can be made by the PNC to allow reference nodes to determine range information to specified devices. Alternately, if capable, the reference nodes, which are constantly "overhearing" conversations in their neighborhood can use start times of transmissions which are published in beacons to infer range information. The PNC uses the range information collected from multiple reference nodes to triangulate the location of the device within the piconet. The more the number of reference nodes used, the more accurate is 
the positioning estimate for a device. The position information is periodically updated by the PNC (by allocating special timeslots for this) to handle device mobility. Since devices are not very dynamic in the environments we are considering, the frequency with which the positioning information is gathered can be low. Greater the frequency with which the positioning is performed, greater the accuracy of the location information with the downside being the increased overhead.

Once device locations are known, the PNC can make more efficient access control decisions for the piconet. We propose enhancing the classical 802.15.3 MAC by allowing the PNC to schedule concurrent parallel non-interfering power-limited transmissions to occur in the piconet. In our model, devices make request for channel access in the same way as in traditional 802.15.3. The PNC uses the information it has about the location of the transmitter and receiver pair to determine the appropriate power needed by the transmitter to reach the receiver. In addition, the PNC can consider other requests for channel access and decide if two or more such requests can be scheduled together without interfering. Two flows are permissible at the same time only if there is complete exclusion between the two flows, i.e.

- Transmission from Source flowA cannot be detected at Receiver $_{\text {flow } B}$

- Transmission from Source flowB cannot be detected at Receiver flow A

- Transmission from Source flowA cannot be detected at Source $_{\text {flowB }}$ and vice versa

- Transmission from Receiver flowA cannot be detected at Receiver flow $B$ and vice versa

The first two rules ensure that the transmitter of a flow will not intefere with the other flow's receiver thereby ensuring receiver exclusion. The third and fourth rules are needed based on the ACK policy used in the piconet. In case of immediate, delayed or implied ACK policy, a source should be able to receive the ACKs being sent by the receiver without inteference from another source nearby and a receiver sending out an ACK should not intefere with data reception at another receiver nearby. In case of a No ACK policy, these rules can be relaxed thereby further improving the possibility of finding candidate parallel flows.

Once the channel allocation has been determined, the PNC publishes this in the periodic beacons to notify the piconet participants. The current 802.15.3 MAC allows the PNC to set a max transmit power for the devices within the piconet. We propose introducing an additional field in the Channel Time Allocation (CTA) status IE (or using the application specific IE) for a CTA request that informs a transmitter of the max transmit power that that device must not exceed. This additional field allows the PNC to schedule multiple transmitters by limiting the max power of each such transmitter. In this work, we are considering symmetric links so the power used by a receiver for sending out ACKs is also limited to the maximum transmit power allowed for that flow as specified by the PNC. Unlike other power control MACs that limit transmit powers to improve power consumption characteristics and interference with other piconets, we are limiting transmit power to minimize interference within the piconet. The accuracy of the positioning information is critical for the PNC to be able to schedule parallel flows without them interfering. A trade-off exists between the overhead incurred in maintaining the positioning information versus the improvement in throughput and increase in the number of admissible flows that can be achieved within the piconet.

We restrict our enhancement to isochronous and asynchronous time allocation requests. Unlike pseudo-static time allocations, these requests can be moved around (position within a superframe) from beacon to beacon. This is an important feature that the PNC exploits to move allocations around so that where ever possible, parallel transmissions can be scheduled. This is necessary in cases where devices exhibit mobility in the environment. For pseudo-static allocations, this is more difficult since these time allocations need to persist across numerous superframe intervals and scheduling parallel such allocations may lead to collisions when devices move leading to inefficient channel usage. For setting transmit powers for transmissions, the PNC takes into account a power margin. This margin determines the excess power that a tranmitter is permitted to use over the minimum required power for a transmission to be successfully received at the receiver. Increasing the margin improves the resiliency to transient interferences in the network but at the cost of inefficient use of the spectrum. At one extreme, with no power margin, any interference will cause the packet to be dropped. At the other extreme, with high power margins, the scheme degrades to a classic 802.15.3 MAC where only one flow can be in progress at any given time slot in the piconet.

\section{Simulation Results}

To study the benefits of our proposed enhancements, we performed simulations using NS2 by extending the 802.15 .3 MAC implementation used for the work in [7]. We considered an area of $40 \mathrm{~m} \mathrm{X} \mathrm{40m} \mathrm{and} 26$ randomly positioned nodes. 6 of these nodes were designated as reference nodes and one of these nodes served as the PNC for the piconet (PNC position was set to $(20,20))$. The line rate used for our simulations was set to $80 \mathrm{Mbps}$ and the all flows in the simulation were $10 \mathrm{Mbps}$ MPEG streams. The received power for a transmission is governed by Friis's law which is dependent on the frequency of transmission. In case of UWB, this implies that we need to integrate over the entire frequency band to arrive at the total received power [18].

$$
\begin{gathered}
d P_{r x}(f)=\frac{c^{2}}{(4 \pi)^{2} r^{2}} \frac{P_{t x}(f) G_{t x}(f) G_{r x}(f)}{f^{2}} \\
P_{R X}=\int_{0}^{\infty} d P_{r x}(f) d f
\end{gathered}
$$

For simplicity sake, We set the lower threshold on the received power to $-50 \mathrm{dBm}$ such that any transmission below that cannot be deciphered (The data rates, transmission ranges 


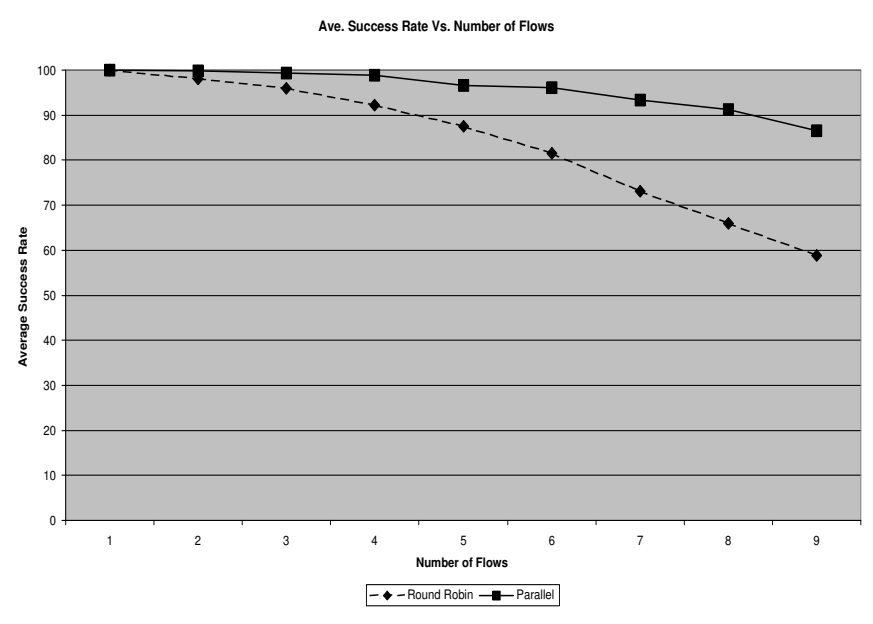

Fig. 4. Success Rate vs. Number of Flows

and receiver sensitivity chosen are conservative compared to commercial products such as [8] with adverstised rates from $55 \mathrm{Mbps}$ to $480 \mathrm{Mbps}$, range from $10 \mathrm{~m}$ to $30 \mathrm{~m}$ and sensitivity of $-80 \mathrm{dbm}$ ). This allows the PNC to determine what transmit power a source device needs to use in order to reach the intended target device (plus margin to compensate for SNR described in our last experiment). This is part of the CTA status IE (or an application specific extension IE) that the PNC uses to grant access to the medium for a device. In addition, we defined a maximum tolerable packet delay for the simulations. Any packet arriving at a destination such that the transmission delay exceeds this threshold is considered a failed job. We analyzed the performance of our proposed approach and compared it against a traditional round robin scheduling scheme. In the round robin scheme, the PNC schedules each Guaranteed Time Slot (GTS) request in a round robin fashion such that each flow gets a time slot in a superframe interval. In all our experiments, transmissions follow an immediate ACK policy within the piconet. We measured the success rate of packet delivery for a flow from its source to the intended destination and use this as a measure of the effectiveness of our proposed approach.

Fig.4 shows the performance comparison between the two schemes when the number of flows in the network is increased. The GTS requests per flow (3ms), the maximum acceptable delay per packet $(50 \mathrm{~ms})$ and the number of nodes $(6$ reference nodes and 20 communicating nodes) in the network were kept constant. The node density of the piconet does not really have a direct effect on the performance of our scheme since only selected nodes during any given time slot can communicate. For this reason, we keep the number of communicating nodes fixed in our experiments and alter the number of flows (upto the maximum that can be supported by the piconet). As the number of flows increase, a round robin type scheme suffers from increased delay between two successive GTS slots for any given flow. This results in queuing delays at each node and the average delay experienced per packet increases. As

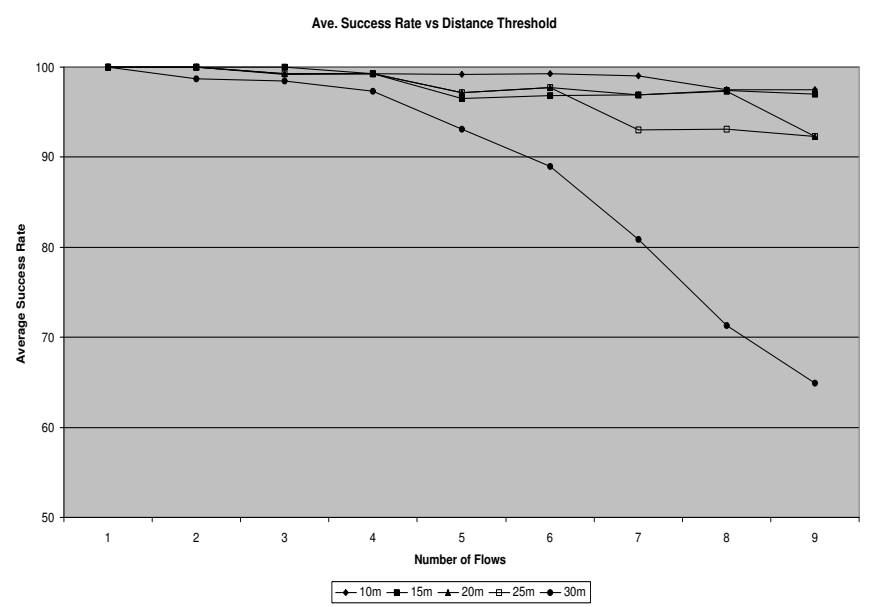

Fig. 5. Success Rate vs. Distance Threshold

a result, the ratio of packets whose delay exceeds the delay thresholds also rises causing them to get dropped. With parallel scheduling, more flows can be scheduled to happen at the same time within the network. This reduces the time between successive GTS slots for a given flow and results in less queuing delays at a node. As a result the ratio of packets that are delivered to a destination within specified delay bounds is higher. In general we found that our scheme performs much better than a round robin scheme under most circumstances. However, there were instances where our scheme performed only slightly better than a round robin approach. In these instances, we found that the flow requests were such that no parallel scheduling was possible (due to the location of the communicating entities) and all flows were awarded channel allocation based on a round robin schedule. We ran experiments by picking source and destination pairs at random such that the distance between them did not exceed a set distance threshold. We ran experiment for distance threshold values of $10 \mathrm{~m}, 15 \mathrm{~m}, 20 \mathrm{~m}, 25 \mathrm{~m}$, and $30 \mathrm{~m}$. As seen in Fig. 5 , with lower distance thresholds, we were seeing much better performance of our scheme while as the distance threshold increased, we had instances where devices at opposite ends of the area were randomly chosen for a flow. In these cases, the power levels of the transmissions were high enough that only one transmission could take place at any given time within the piconet. In general, the more localized the communication within a piconet, the better our scheme performs in comparison to the round-robin approach.

Fig.6 shows the performance comparison when we keep the number of flows in the network (6 flows), number of nodes and the maximum acceptable delay per packet constant and increase the GTS. The lower the GTS, the more beacon packets there are in the network due to the shorter superframe intervals. However, this reduces the average queuing at the nodes since each node gets frequent opportunities to transmit. As the GTS increases, the success rate stays more or less unchanged with corresponding decrease in the number of 


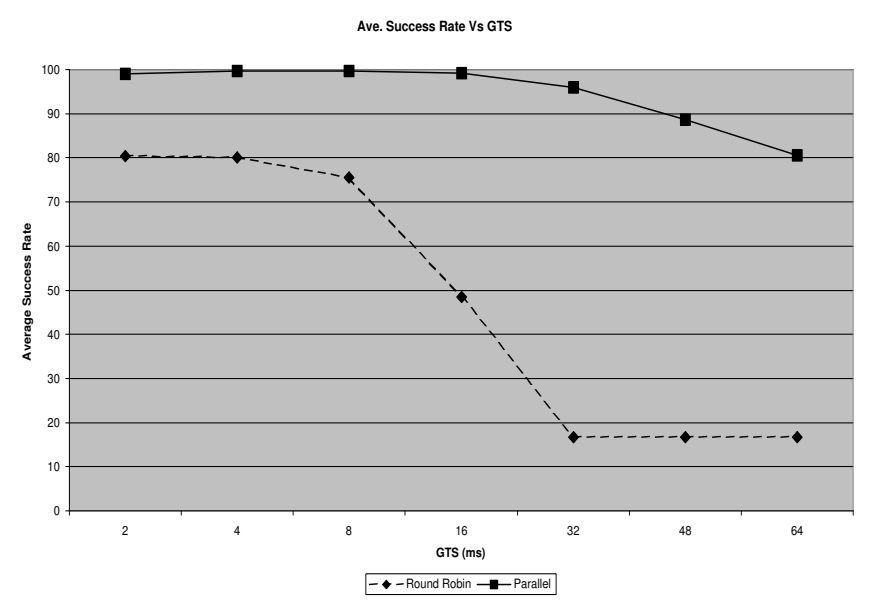

Fig. 6. Success Rate vs. GTS

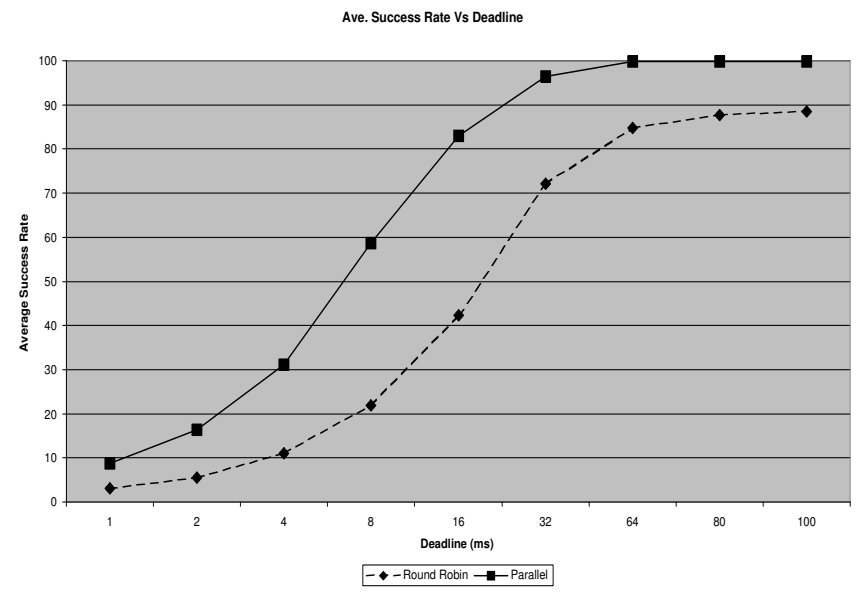

Fig. 7. Success Rate vs. Deadline

beacons. It can be argued that even with low GTS allocations, the number of beacon packets can be minimized by having multiple GTS allocations within a single superframe for the same flow. For very high GTS requests, the success rate can fall significantly (in some cases to $100 \%$ failure) since packet deadlines cannot be met by a flow because a previous flow took up all the time.

Fig.7 shows the effects of changing the maximum acceptable packet delays while keeping the number of flows and the GTS per flow constant. For low values, both schemes suffer from excessive packet losses due to queuing delays at the nodes. As the acceptable delay increases, the performance of both schemes improves. Even for relatively low delay thresholds, parallel scheduling outperforms a traditional round robin scheduling mechanism. By exploiting spatial location and power control, our scheme enables multiple flows to be scheduled in parallel thereby decreasing the time between any two GTS allocations for a given flow. This translates to lower delays experienced by the data packets at the nodes and hence the better performance.

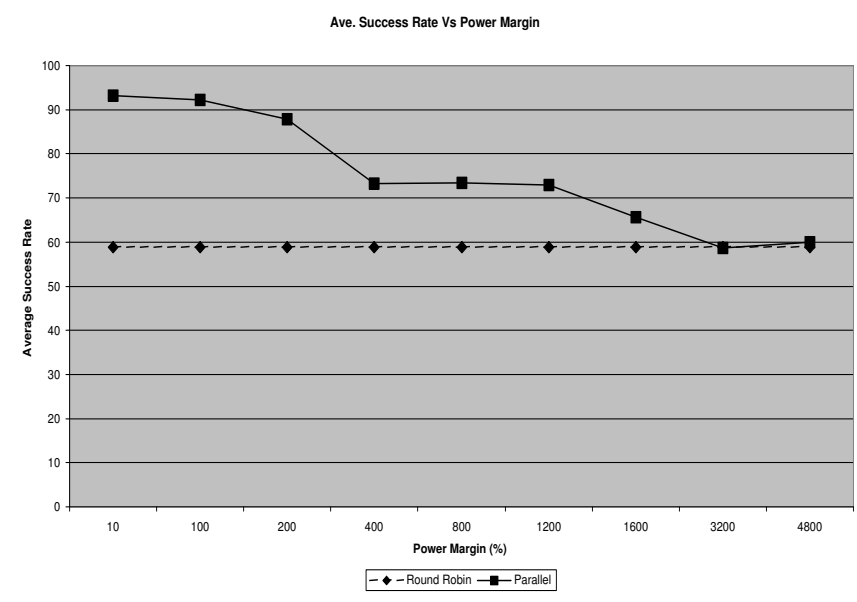

Fig. 8. Success Rate vs. Power Margin

Fig.8 shows the impact of increasing the power margin for parallel transmissions. In our model, the PNC sets the maximum power that a transmitter can use on a per flow basis. Ideally, this value would be based on the minimum power required for a transmission from that transmitter to be successfully received at the receiver (plus a small power margin to compensate for SNR). This can be estimated by the PNC using the distance between the transmitter and receiver and channel status that can be gathered using interference mitigation mechanisms built into 802.15.3 MAC (such as a PNC channel scan or a remote scan request and collection of the channel status from the devices). In this experiment, we keep the number of flows (6 flows), packet arrival deadline (50 $\mathrm{ms})$ and time request per flow $(3 \mathrm{~ms})$ constant and vary the power margin that the PNC allows for parallel transmissions. A higher power margin for a flow implies that the possible maximum transmission power for that flow is higher (but still within the maximum transmission power allowed for that piconet which is set by the PNC and which determines the extent of the piconet). This implies that in order for flows to be scheduled on the same time slots, they need to be sufficiently far enough that they will not interfere with each other. For high power margins allowed by the PNC, there is less potential for finding such flows due to the limited size of the piconet and our scheme starts to behave like a traditional round robin scheduling with the exclusion region spanning the whole piconet. For lower power margins, we see that there are still flows that are sufficiently far enough from each other that they can be scheduled for the same time slots without interfering. A lower power margin also has the benefit that the power consumed by the transmissions within a piconet is lower. The risk, however, is that lower the margin, the lower the resistance the transmission has to external interference. In our approach, the power margins are used to compensate for low SNR. In a noisy environment, the power margins are made sufficiently high (and as a result, there is less possibility for parallelism) so that the transmitter 
can transmit at a high enough power to be able to compensate. In the worst case, the transmitter will transmit at close to the maximum power possible for the piconet thereby becoming more like the traditional 802.15.3 MAC. In the best case, the noise is low enough that we can use a very low power margin (close to 0) to achieve maximum utilization of the channel.

\section{CONCLUSION}

In this paper, we are proposing extending the traditional 802.15.3 MAC for UWB networks to take into consideration relative location of communicating entities while performing channel scheduling for the piconet. The spatial location of devices within a network is used in combination with a power control mechanism to allow pairs of devices to communicate in parallel within a piconet. Rather than enforcing an exclusion region that spans the whole piconet, our mechanism allows multiple devices to communicate as long as any such communication does not adversely affect another. The PNC uses a power control scheme that limits the maximum transmit power used by a communicating pair based on their location which in addition to restricting the exclusion region around them also improves the power consumption characteristics of the network. We have presented simulation studies comparing our enhancements to a traditional round robin type scheduling mechanism and our scheme shows much better network throughput under various scenarios considered.

We are currently considering the effects of device mobility within the piconet as it pertains to our proposed enhancements. As devices move (albeit relatively slowly in indoor environments), the PNC needs to allocate more time slots for the gathering of location information. This decreases the available time for actual data transmission. A tradeoff exists between the benefits of parallel transmissions versus costs incurred in gathering location information. Several facets such as the accuracy of location information needed, number of flows, number of nodes, speed of motion, acceptable packet losses due to collisions etc. need to be considered. We are considering enhancements to allow a PNC to decide when to use parallel transmissions and when to fall back to a more traditional scheduling mechanism dynamically. Also, we need to further investigate mechanisms to allow the PNC to be able to dynamically adapt the power margins based on the SNR observed at the receivers due to varying conditions.

\section{ACKNOWLEDGEMENT}

The authors would like to acknowledge the valuable comments and suggestions provided by Dr. Krishna Sivalingam of the University of Maryland, Baltimore County.

\section{REFERENCES}

[1] Bluetooth Core specification V2.0, World Wide Web, http://www . bluetooth.org/spec/.

[2] IEEE 802.15 WPAN High Rate Alternative PHY Task Group 3a (TG3a), http://www. ieee802.org/15/pub/TG3a.html.

[3] Part 15.3: Wireless medium access control (MAC) and physical layer (PHY) specifications for high rate wireless perosnal area networks (WPAN), IEEE 802.15.3 Working Group, Draft P802.15.3/D16, 2003.
[4] DS-UWB Physical Layer Submission to 802.15 Task Group 3a, ftp: //ieee:wireless@ftp.802wirelessworld.com/15/04/ 15-04-0137-03-003a-merger2-proposal-ds-uwb-update. doc.

[5] Multi-band OFDM Physical Layer Proposal for IEEE 802.15 Task Group 3a, ftp://ieee:wirelesseftp. 802wirelessworld.com/15/Archive/2003/Jul03/ 03268r3P 802-15_TG3a-Multi-band-CFP-Document.doc.

[6] WiMedia Alliance, http://www.wimedia.org.

[7] Performance and Simulation Analysis of 802.15 .3 QoS, http://grouper.ieee.org/groups/802/ 15/pub/2002/Jul02/02297r1P802-15_TG3_ Performance-Analysis-of-MAC-and-QoS.ppt.

[8] UBLink Chipset, Wisair Ltd., http://www.wisair.com.

[9] J.-Y. L. Boudec, R. Merz, B. Radunovic, and J. Widmer. Dcc-mac: A decentralized mac protocol for 802.15.4a-like uwb mobile ad-hoc networks based on dynamic channel coding. In Broadnets'04, October 2004.

[10] W. C. Chung and D. S. Ha. An accurate ultra wideband (uwb) ranging for precision asset location. In IEEE Conference on Ultra Wideband Systems and Technologies (UWBST'03), Reston, VA, USA, November 2003.

[11] N. S. Correal, S. Kyperountas, Q. Shi, and M. Welborn. An uwb relative location system. In IEEE Conference on Ultra Wideband Systems and Technologies (UWBST'03), Reston, VA, USA, November 2003.

[12] F. Cuomo, C. Martello, A. Baiocchi, and F. Capriotti. Radio resource sharing for ad hoc networking with uwb. In IEEE Journal on Selected Areas in Communications, volume 20.

[13] R. Fleming, C. Kushner, G. Roberts, and U. Nandiwada. Rapid acquisition for ultra-wideband localizers. In IEEE Conference on Ultra Wideband Systems and Technologies (UWBST'02), Baltimore, MD, USA, May 2002.

[14] R. J. Fontana and S. J. Gunderson. Ultra-wideband precision asset location system. In IEEE Conference on Ultra Wideband Systems and Technologies (UWBST'02), Baltimore, MD, USA, May 2002.

[15] S. Kolenchery, J. K. Townsend, and J. A. Freebersyser. A novel impulse radio network for tactical military wireless communications. In IEEE Military Communications Conference (MILCOM'98), 1998.

[16] J.-Y. Lee and R. A. Scholtz. Ranging in a dense multipath environment using an uwb radio link. In IEEE Journal on Selected Areas in Communications, volume 20

[17] K. Lu, D. Wu, Y. Fang, and R. C. Qiu. On medium access control for high data rate ultra-wideband ad hoc networks. In IEEE Wireless Networking and Communications Conference WCNC'05 (to appear), March 2005.

[18] H. G. Schantz. Introduction to ultra-wideband antennas. In IEEE Conference on Ultra Wideband Systems and Technologies (UWBST'03), Reston, VA, USA, November 2003.

[19] M. Z. Win and R. A. Scholtz. Ultra wide bandwidth timehopping spreadspectrum impulse radio for wireless multiple access communications. In IEEE Transactions on Communications, April 2000. 